XLV.-On the Estimation of Compound Ethers in Wine.

$$
\text { By A. Dupré, Ph.D. }
$$

BERTHELOT was, I believe, the first who endeavoured to estimate the amount of compound ethers in wine. He did so by means of a process of titration, also devised by himself. The process consisted, firstly, in estimating the total amount of free acid in the wine by means of a standard solution of baryta; and, secondly, in heating 50 c.c. of the same wine in a closed tube, with a known excess of the same baryta-water, after which the amount of baryta still left unsaturated was determined. During the heating, the compound ethers are decomposed into alcohol and acid, which latter neutralizes part of the baryta. A wine containing any compound ethers will, therefore, neutralize more alkali by being heated with it than it will in the cold; and this excess of alkali neutralized is an exact measure of the amount of acid present as compound ether, and indirectly a measure of its equivalent of alcohol.

With pure compound ethers, or their aqueous or alcoholic solutions, this process gives admirable results, but it is only in exceptional cases applicable to wine, inasmuch as they nearly always contain substances, besides the compound ethers, which neutralize alkalis when heated with them. It is of course totally useless in all cases; where the wine contains an appreciable amount of sugar. The plan proposed in the following pages is free from this objection, whilst it gives, I believe, equally accurate results, even in cases where Berthelot's process is applicable.

The compound ethers present in wine may be divided into two classes, namely: those which are readily volatilized without decomposition, like acetic ether, and those which are not volatile without decomposition, like tartaric ether. If therefore a wine be carefully distilled, part of its ether will pass over into the distillate, part will remain behind in the residue. The ether in the distillate may be estimated by Berthelat's plan, there being nothing present to interfere with it. I am in the habit of using for this purpose a deci-normal solution of soda*, and

* A deci-normal solution of soda contains $\frac{1}{10}$ of an equivalent of caustic soda in 1 litre solution.

VOL. XX. 
heating the ether and alkali in a water-bath, in a long-necked assay flask, closed by a well fitting caoutchouc-stopper, tied down. The heating may be continued from one to five hours without appreciable difference in the result. The ether in the residue is decomposed by heating with the alkali, and the alcohol produced is distilled over and estimated. We thus obtain the amount of acid present in the volatile ethers, and the amount of alcohol present in the fixed ether. By calculation it is of course easy to substitute one for the other in either.

I have satisfied myself by numerous experiments, that if a wine be carefully distilled-from one-half to four-fifths, or even more, being driven over-and the distillate be then again added to the residue in the retort, the amount; of free acid present is not appreciably altered by the heating; thereby proving that none of the compound ethers have been decomposed, otherwise the amount of free acid would have increased.

The following examples will show this :-

Rauenthaler, 1862.

\section{RhINe Wines.}

50 c.c. wine required $38 \cdot 0$ c.c. deci-normal soda.

300 c.c. of same wine were submitted to distillation, threefourths driven over; on adding the distillate to the residue 50 c.c. of the mixture required $37 \cdot 8$ c.c. d. n. soda.

Rauenthaler, 1864.

50 c.c. wine required $55 \cdot 5$ c.c. $d$. n. soda.

50 c.c. wine distilled in water-bath; the distillate added to the residue required $55 \cdot 3$ c.c. $d . n$, soda.

\section{Hattenheimer, 1862.}

50 c.c. wine required 40 c.c. d. n. soda.

50 c.c. wine, distilled over an argand burner, more than onehalf driven over; the distillate added to the residue, required 39.9 c.c. d. $n$. soda.

Sherry, about fifty years in bottle.

50 c.c. wine required $42 \cdot 7$ c.c. d. n. soda.

50 c.c. wine, distilled nearly to dryness in sand-bath, and water added to the residue, which was again distilled nearly to dryness; the distillates added to the residue, required 42 c.c. d. n. soda. 
Madeira, bottled in 1817 .

50 c.c. wine required $39 \cdot 7$ c.c. d. $n$. soda.

50 c.c. wine, distilled nearly to dryness in salt-water bath; the distillate and residue required $38 \cdot 7$ c.c. d. n. soda.

It will be seen that, in all cases, the amount of free acid is but slightly altered by distillation, and contrary to what might have been expected, it shows rather a diminution than an increase. Indeed, if the residue in the retort be heated for too long a time, or too strongly, its acidity is almost destroyed, without much more acid passing into the distillate.

As, however, the last traces of free alcohol are not readily distilled off from a wine without danger of decomposing some of its constituents, I prefer to estimate the volatile and fixed ethers in two different quantities:-

1st. From 250 c.c. wine about 200 c.c. are distilled off, and the distillate is made up to 250 c.c. In 100 c.c. of this the free acid is determined by a standard solution of soda, and a second 100 c.c. is heated with a known excess of alkali, as previously described.

2nd. Another 250 c.c. of the wine are evaporated on a waterbath to about 40 c.c. The residue is put into a flask, rendered strongly alkaline by caustic potash or soda, and about threefourths of it are distilled over. During this distillation the liquid is very apt to froth over, even after addition of tannin, and the distillation must, therefore, be conducted with care. This first distillate, generally strongly alkaline, is rendered slightly acid by sulphuric acid, and again distilled, about 20 c.c. being driven over. These 20 c.c. will contain all the alcohol originally present in the 250 c.c. wine as fixed ether; they contain no appreciable quantity of anything else.

At first I estimated the amount of alcohol in these 20 c.c. by means of their specific gravity, and with the requisite amount of care accurate results may thus be obtained. Inasmuch, howover, as the spirit obtained is exceedingly weak, it is very difficult to avoid an error large enough to vitiate the whole result; and, therefore, since the publication of Mr. Chapman's paper on limited oxidation, I estimate the alcohol by converting. it into acetic acid, which latter can be accurately and easily determined. The alcohol may also be estimated with tolerable accuracy by means of Geis sler's vaporimeter.

For the purpose of oxidation, the 20 c.c. distillate are trans- 
ferred to a small strong flask (I make use of the small assay flasks). 10 c.c. of bichromate solution are added, and the flask, after having been securely closed by an india-rubber stopper, tied down firmly, is suspended for one or two hours in a waterbath kept boiling. The bichromate solution contains 10 per cent. bichromate, and enough sulphuric acid to be capable of just forming chrome-alum if all the chromic acid were reduced to chromic oxide, as recommended by Mr. Chapmann. 10 c.c. of such a solution are capable of oxidizing about $0.2 \mathrm{grm}$. alcohol into acetic acid.

After the flask has been heated for about two hours, it is taken out of the water-bath, cooled, opened, and after the addition of some sulphuric acid, the excess of bichromate left is reduced by zinc. The green solution is next transferred to a small retort, and the acetic acid contained in it is distilled over. This distillation may be conducted either in an oil-bath or over an argand burner, and it is as well to place some pieces of broken tobacco-pipe in the retort to prevent bumping. I have found no difficulty in distilling over all the acetic acid, especially if, after the distillation has been continued nearly to dryness, some more water be added to the residue, and the distillation resumed, repeating the same process two or three times.

The acetic acid in these distillates is then estimated by means of a deci-normal solution of caustic soda, each c.c. of which neutralizes $0.006 \mathrm{grm} . \bar{A}$, and indicates, therefore, $0.0046 \mathrm{grm}$. alcohol. The total amount of alcohol thus found, divided by $2 \cdot 5$, gives the percentage of alcohol contained in the fixed ethers of the wine.*

* I have used the same method very successfully for estimating the small amount of alcohol excreted through the kidney, after moderately large doses if wine or spirit bad been taken. The total amount of urine passed during the 24 hours following the taking of the wine or spirit was collected, and rendered slightly acid; some tannin was added; and the liquid was distilled, about $\frac{1}{3}$ being driven over. This first distillate was then made slightly alkaline, and again submitted to distillation, and this re-distillation repeated, $\frac{1}{3}$ being distilled over each time, until the final distillate amounted to about 20 c.e., in which the alcohol was determined as above. All the distillations of alcoholic liquids, as well as those of acetic acid, were conducted with an apparatus in which the retort, as well as the receiver, was connected, air.tight, with the condenser, the receiver being furnished with a safety-tube closed by mercury. The enclosed air was thus, to a certain extent, allowed to expand or contract, whilst, as long as the distillation is proceeding quietly, the receiver is closed entirely, and all evaporation thus prevented. I was thus enabled to distil either moderately 
If a little attention be given to the distillation, it will be found that the distillate containing the acetic acid is quite free from sulphuric acid, and its acidity, as measured by titration, is, therefore, a correct measure of the acetic acid it contains. But even if some sulphuric acid has passed into the distillate, it is nevertheless easy to estimate the acetic acid by titration. We have simply either to divide the distillate accurately into two equal parts, estimating the acidity of one-half directly, whilst the second half is evaporated on a water-bath, and the remaining acidity then determined,- - which, being subtracted from the amount found in the first half, leaves the acidity due to the acetic acid; -or, secondly, after having carefully determined the amount of alkali necessary to neutralize the entire distillate, an equal amount of deci-normal sulphuric acid is added, and the whole evaporated to dryness on a water-bath. If the distillate consisted only of acetic acid, the dry residue, on being dissolved in water, will be found to be neutral. Any acidity remaining will be due to sulphuric acid, and has to be subtracted from that first found, to obtain the true measure of the acetic acid.

It will scarcely be necessary to add that if an amount of alcohol be found equal, or approximately equal, to the total amount capable of being oxidized by the bichromate, a second estimation has to be made with more bichromate or less alcoholic solution. I have not, however, as yet, met with any wine which, from 250 c.c., yielded even half the amount of alcohol from its fixed ethers that could be oxidized by 10 c.c. of the above bichromate solution, nor is it at all likely that such a wine exists.

In the following tables, I give the various analytical results upon which the foregoing method is based.

1st. All free alcohol may be expelled by partial evaporation of the liquid on a water-bath.

In half a litre of spirit of 13 per cent. by weight, $20 \mathrm{grm}$. canesugar were dissolved, and the whole was evaporated on a waterbath to 60 c.c. These 60 c.c. were submitted to distillation, 25 c.c. distillate were collected, which, at a temperature of $155^{\circ}$, had a spec. gr. of 999.99 , water at the same temperature being taken at 1000 .

strong or very diluted spirits, even four or five times, without any appreciable loss of alcohol. 
2nd. No acid tartaric ether is decomposed or volatilized during such evaporation.

A dilute solution of acid tartaric ether in spirit of 10 per cent. was prepared, containing in 20 c.c. an amount of acid, neutralized in the ether, equivalent to $10 \cdot 1$ c.c. d. n. soda. The free acid present in 20 c.c. of the mixture required $28 \cdot 2$ c.c. d. n. soda. Four quantities of 20 c.c. each were next put into small porcelain dishes, and heated on a water-bath; the first was allowed to evaporate to $\frac{1}{4}$, the second to $\frac{1}{10}$, the third just to dryness, and the fourth was heated on the water-bath for an hour after it had become dry; the free acid in all the samples was then again determined.

20 c.c. of the original mixture required $28 \cdot 2$ c.c. d. n. soda

The residue from the first dish

$\begin{array}{llll}\Rightarrow & 28 \cdot 2 & " & " \\ " & 28 \cdot 4 & " & " \\ " & 28 \cdot 4 & " & "\end{array}$

thus showing an "exceedingly slight alteration only" had taken place.

3rd. Two solutions of pure tartaric ether in alcohol being made, the amount of alcohol present as ether in each was estimated by titration; to the weaker of the two, 4 per cent. canesugar were next added, and the alcohol present in the ether was then also estimated by the process given above. For this purpose, $\frac{1}{2}$ litre of each solution was evaporated on a water-bath to 60 c.c.; this residue was rendered strongly alkaline and distilled; and the first distillate after being acidified was re-distilled, 28.5 c.c. of the second distillate being collected in each case. These second distillates had, at a temperature of $155^{\circ}$, a sp. gr. of 997.50 and 998.30 , showing a strength of 1.34 per cent. and 0.91 per cent. by weight respectively, which, calculated upon the $\frac{1}{2}$ litre taken, gives 0.0760 and 0.0520 per cent. alcohol present as ether in the two mixtures. Titration had given 0.08004 and 0.05428 per cent. We have therefore-

By titration, By evaporation, \&c.

$\begin{array}{llll}\text { In 1st mixture........ } & 0.08004 & 0.0760 \\ , \text { 2nd }, \quad \ldots \ldots \ldots & 0.05428 & 0.0520\end{array}$

Lastly, two very dilute spirits were prepared, by carefully mixing the requisite weights of absolute alcohol and water, containing in 20 c.c. 0.1 grm. and 0.025 grm. of absolute alcohol. 
In 20 c.c. of these mixtures the alcohol was oxidized by bichromate solution, in the manner before described, and the resulting acetic acid estimated by titration. The distillate from the stronger mixture required $20 \cdot 1$ c.c. d. n. soda solution for neutralization, equivalent to $0.0924 \mathrm{grm}$. alcohol; the distillate from the weaker mixture required 5.5 c.c. d. n. soda, equivalent to $0.0253 \mathrm{grm}$. alcohol.

1st. Alcohol taken 0.1 grm. found 0.0924 grm. 2nd. " $0.025, \quad, 0.0253$,

\section{Application of the Method to Wine.}

In the case of light wines, containing but little or no sugar and extractive, it is sufficient, in estimating the alcohol of their fixed ether, simply to evaporate them to about $\frac{1}{5}$ th or $\frac{1}{10}$ th their bulk; with strong wines containing much sugar it is, however, advisable, either to dilute them considerably before evaporation, or, after they have been evaporated to $\frac{1}{6}$, to dilute the residue with 100 c.c. or 200 c.c. water, and once more evaporate down to about 40 c.c. In either case the residue is distilled with alkali, \&c., \&c., as previously described. The alcohol in the second distillate may be estimated by its specific gravity, using Geissler's vaporimeter, or, most accurately, by oxidation and estimation of the acetic acid formed. If care be taken, all three methods give the same result.

Berthelot has given a formula by means of which the total amount of alcohol contained as ether in any wine, when etherification is complete, can be calculated from the known amount of free acid and alcohol present. For the sake of comparison I have added this theoretical number to each analysis; in young wines the amount of ether found should fall below this theoretical value, whilst in older wines the two values should agree.

\section{RHINE WINES.}

Rauenthaler, 1864.-18s. per dozen.

Alcohol................ 7.44 per cent., by wt. Total free fixed acid calculated as $\overline{\mathrm{T}}$. . $0 \cdot 674$ per cent. " volatile $, \quad \quad, \quad \bar{A} . .0 .118$ per cent. Total dry residue............. 2.207 per cent. Sugar................... traces. 


\section{Estimation of Alcohol in fixed Ethers.}

a. Alcohol estimated by specific gravity.

1 litre of wine was distilled in a salt water-bath; 900 c.c. distilled over; the residue was rendered strongly alkaline, and the distillation proceeded with; 60 c.c. were collected, which, at a temperature of $15 \cdot 5^{\circ} \mathrm{C}$, had a sp. gr. of $999 \cdot 14$, showing 0.45 per cent. by weight of alcohol, or 0.027 per cent. of original wine.

300 c.c. wine distilled over an argand lamp; 200 c.c. passed over, residue treated as above, but the first distillate was rendered acid and again distilled; 25 c.c. off second distillate collected, which had, at a temperature of $15 \cdot 5^{\circ} \mathrm{C}$., a sp. gr. of $999 \cdot 45$, showing 0.29 per cent. alcohol, or 0.024 per cent. of original wine.

$b$. Alcohol estimated by oxidation.

250 c.c. wine evaporated on a water-bath to 50 c.c.; residue rendered alkaline and distilled; first distillate acidified and redistilled; second distillate of 20 c.c. oxidized by bichromate solution as before described. Acetic acid obtained on distillation, neutralized $14 \cdot 2$ c.c. d. n. soda, equivalent to $0.0653 \mathrm{grm}$. alcohol, or 0.0261 per cent. of wine.

100 c.c. wine, evaporated on water-bath to 25 c.c., residue treated as above. $\bar{A}$ obtained neutralized 6.0 c.c. d. n. soda, equivalentt to 0.0276 per cent. alcohol in wine.

250 c.c. wine, evaporated to 25 c.c. $\overline{\mathrm{A}}$ obtained neutralized $12 \cdot 6$ c.c. d. n. soda, equivalent to $0.05796 \mathrm{grm}$. alcohol, or 0.0232 per cent. of wine.

We have therefore alcohol present in fixed ethers-

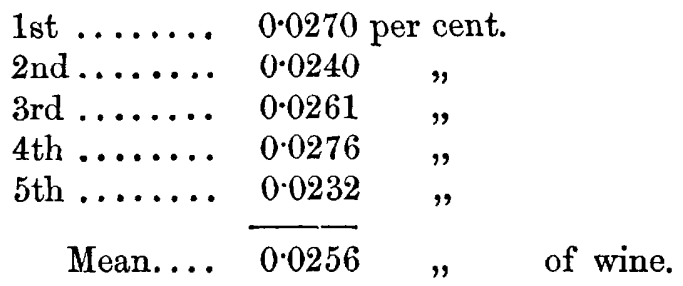

Alcohol in volatile ethers, found by titration............... 0.0175 per cent.

Total alcohol in ethers found..... 0.0431 "

Total alcohol in ethers calculated .. 0.0581 " 
Rauenthaler, 1862.-54s. per dozen.

Alcohol ............ 8.83 per cent. by weight.

Free fixed acid, as $\overline{\mathrm{T}} \ldots \ldots .0 .445$

Free volatile acid as $\overline{\mathrm{A}} \ldots . .0 .178$

Total dry residue ....... 1.867

Sugar .............. 0.062

"

Fixed Ethers.

a. Alcohol estimated by vaporimeter.

Half a litre of wine evaporated on water-bath to $125^{\circ}$ c.c.; residue treated as above; second distillate amounted to 25 c.c., showing by vaporimeter 0.45 per cent. alcohol by weight, equal to 0.0225 per cent. of wine.

$b$. Alcohol estimated by oxidation.

300 c.c. wine evaporated to 20 c.c. ; residue treated as above. $\bar{A}$ obtained neutralized $14 \cdot 5$ c.c. d. n. soda, equivalent to 0.0667 grm. alcohol, or 0.0222 per cent. of wine.

250 c.c. wine evaporated to 50 c.c. ; residue treated as above. $\bar{A}$ obtained neutralized $11 \cdot 3$ c.c. d. n. soda, equivalent to $0.05198 \mathrm{grm}$. alcohol, or 0.0208 per cent. of wine.

$$
\begin{array}{rr}
\text { 1st ........ } & 0.0225 \text { per cent. } \\
\text { 2nd ........ } & 0.0222 \quad " \\
\text { 3rd ....... } & 0.0208 \quad " \\
\text { Mean.... } & 0.0218 \quad,
\end{array}
$$

Alcohol in volatile ethers by titration. . 0.02392 per cent.

Total alcohol in ethers found ....... 0.04572 ",

Total alcohol in ethers calculated .... 0.045957 ",

Hattenheimer, 1862.-40s. per dozen.

Alcohol ........... 9.97 per cent. by weight.

Free fixed acid as $\overline{\mathrm{T}} \ldots \ldots .0 .525$

Free volatile acid as $\bar{A} \ldots .0067$

Total dry residue ....... $2 \cdot 235$

Sugar ............... 0.017

$"$
$"$
"

Fixed Ethers.

a. Alcohol estimated by specific gravity.

300 c.c. wine distilled; two-thirds distilled over; residue treated 
as above; second distillate amounted to 25 c.c., having a specific gravity of 999.58 , showing 0.22 per cent. alcohol, or 0.0183 per cent. of wine.

b. Alcohol estimated by vaporimeter.

The above 25 c.c. gave by vaporimeter 0.25 per cent., or 0.0208 per cent of wine.

c. Alcohol estimated by oxidation.

250 c.c. wine evaporated to 40 c.c. $\bar{A}$ obtained neutralized 11.4 c.c d. n. soda, equivalent to $0.05244 \mathrm{grm}$. alcohol, or 0.0209 per cent of wine.

250 c.c. wine evaporated to 30 c.e. $\bar{A}$ obtained neutralized, $11 \cdot 2$ c.c. d. $n$ soda, equivalent to 0.05152 grm. alcohol, or 0.0206 per cent of wine.

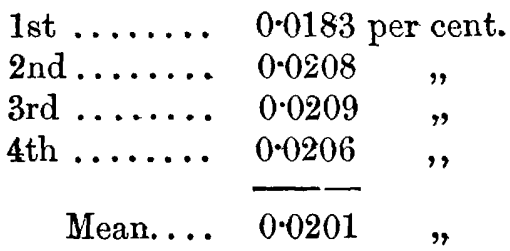

Alcohol in volatile ethers by titration .. 0.0253 per cent. Total alcohol in ethers found ......... 0.0454 " Total alcohol in ethers calculated ..... 0.0521 ",

Steinberg Cabinet, 1858.-120s. per dozen. Alcohol.............. 9.74 per cent. Free fixed acid as $\overline{\mathrm{T}} \ldots . . .0004113$, Free volatile acid as $\overline{\mathrm{A}} \ldots . .0 .1305 \quad$ " Total dry residue ....... $2 \cdot 073 \quad$ " Sugar ............. none.

\section{Fixed Ethers.}

300 c.c. wine evaporated to 20 c.c.; residue treated as usual. $\bar{A}$ obtained neutralized 14.5 c.c. d. n. soda, equivalent to 0.0667 grm. alcohol, or 0.0222 per eent. of wine.

250 c.c. wine evaporated to 50 c.c. $\overline{\mathrm{A}}$ obtained neutralized 13.4 c.c. d. n. soda, equivalent to $0.06164 \mathrm{grm}$. alcohol, or 0.0246 per cent. of wine. 
COMPOUND ETHERS IN WINE.

1st ....... 0.0222 per cent.

2nd ....... $0.0246 \quad$,

Mean.... $\overline{0.0234}$,

Alcohol in volatile ethers by titration. . 0.02944 per cent. Total alcohol in ethers found ....... 0.0528 , Total alcohol in ethers calculated .... 0.0499 ",

Light Claret, 1865.-15s. per dozen.

Alcohol............... 9.05 per cent.

Free fixed acid as $\overline{\mathrm{T}} \ldots \ldots \ldots .0 .338$,

Free volatile acid as $\bar{A} \ldots \ldots .0 .222$,

Total dry residue ........ $2 \cdot 167$ "

Sugar .............. 0.047 "

\section{Fixed Ethers.}

250 c.c. wine evaporated to 15 c.c. $\vec{A}$ obtained neutralized $7 \cdot 4$ c.c. d. n. soda, equivalent to 0.03404 grm. alcohol, or 0.0136 per cent. of wine.

250 c.c. wine evaporated to 40 c.c. $\overline{\mathrm{A}}$ obtained neutralized, $8 \cdot 3$ c.c. d. n. soda, equivalent to $0 \cdot 03818 \mathrm{grm}$. alcohol, or $0 \cdot 0152$ per cent.

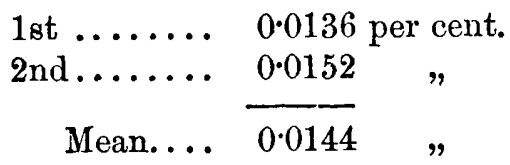

Alcohol in volatile ethers ....... 0.0170 per cent.

Total alcohol in ethers found...... 0.03140 "

Total alcohol in ethers calculated.. 0.05056 "

Cape Wine.-18s. per dozen.

Alcohol ............. 24.1 per cent. by wt.

Free fixed acid as $\overline{\mathrm{T}} \ldots \ldots . .00 .2244 \quad$,

$\begin{array}{llll}\text { Free volatile acid as A } & \ldots . . & 0.1470 \quad \text {, }\end{array}$

Total dry residue........ $6 \cdot 18 \quad$ ",

Sugar.............. 2.88 "

250 c.c. wine, evaporated to 40 c.c. $\bar{A}$ obtained neutralized 
15.9 c.c. d. $n$. soda, equivalent to 0.07314 grm. alcohol, or 0.0292 per cent. of wine.

250 c.c. wine, evaporated to 25 c.c. $\bar{A}$ obtained neutralized 15.9 c.c. d. n. soda, equivalent to $0.07314 \mathrm{grm}$. alcohol, or 0.0292 per cent. of wine.

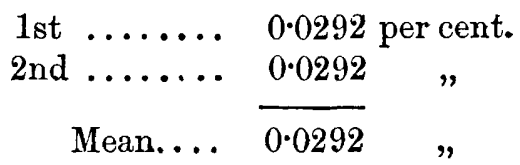

Alcohol in volatile ethers ....... 0.03348 per cent.

Total alcohol in ethers found .... 0.0627 ,

Total alcohol in ethers calculated. . 0.08276 ",

\section{Port.-1864.}

Alcohol ............... 18.56 per cent.

Free fixed acid as $\overline{\mathrm{T}} \ldots \ldots \ldots . .0 .0 .3075$,

Free volatile acid as $\overline{\mathrm{A}}$....... 0.0840 ,

Total dry residue ......... 7.305 ,

Sugar ............... 4... 405 ,

\section{Fixed Ethers.}

250 c.c. wine, evaporated to 40 c.c.; 200 c.c. water added, and again evaporated to 40 c.c.; residue then treated as before described. $\bar{A}$ obtained neutralized 16.4 c.c. d. n. soda, equivalent to $0.07544 \mathrm{grm}$. alcohol, or 0.030176 per cent. of the wine.

Alcohol in volatile ethers ....... 0.01288 per cent. Total alcohol in ethers found .... 0.043056 "

Total alcohol in ethers calculated. . 0.062023"

I am now engaged in the examination of a great variety of wines, and hope in a future communication to be able to show the influence which age, climate, addition of alcohol, etc., etc., have on the quantity and quality of the compound ethers of the wine.

Laboratory, Westminster Hospital, September 10, 1867. 